\title{
Ubiquity and Pervasivity: On the Technological Mediation of (Mobile) Everyday Life
}

\author{
Giuseppina Pellegrino \\ University of Calabria, Department of Sociology and Political Science \\ Italy, gpellegrinous@yahoo.com,g.pellegrino@unical.it
}

\begin{abstract}
This paper aims to contribute to the debate about relationships between technology and society, or technology in society, starting from the categories of ubiquity and pervasivity. The analysis will try to understand ubiquitous/pervasive computing as a new frontier in contemporary movements of computerization [cf. Iacono and Kling, 2001], framing it in the interrelationships between different interests expressed in public discourse. Convergence in hi-tech industry and technological artefacts emerging from organizational and socio-cultural arrangements put forward the categories of ubiquity and pervasivity as key-words in design, functionality and perception of technological artefacts. The concept of ubiquity focuses on both the mobility and the pervasivity/embeddedness of technological artefacts that support the emergence of mobile Internetworking in a mobile society. Mobility and a set of affiliated concepts (e.g. miniaturization, portability, integration) constitute the main discursive frame in mobile and ubiquitous computing. Different layers of public discourse emerge as pertinent to this technology: a technology-driven and a social software perspective, both featured in the media discourse. All of them frame, eventually, inclusionary and exclusionary patterns of sociotechnical action, emerging from different politics of signification.
\end{abstract}

Keywords: ubiquity, mobility, discursive frames, mobile and ubiquitous computing, computerization movements

\section{Introduction}

This paper draws from the analysis of emerging concepts like mobility and mobile society, ubiquitous and mobile computing, pervasive nomadic information environments, to understand how they constitute a relevant dimension to frame, imagine and represent change and transformation in contemporary everyday life. In

Please use the following format when citing this chapter:

Pellegrino, G., 2006, in IFIP International Federation for Information Processing, Volume 223, Social Informatics: An Information Society for All? In Remembrance of Rob Kling, eds. Berleur, J., Numinen, M. I., Impagliazzo, J., (Boston: Springer), pp. 133-144. 
this sense, mobile and ubiquitous computing is an open laboratory where links between the designers' work, new sociotechnical arrays, social imagery concerning technology in everyday life are experienced, enacted and drawn. Ubiquity and pervasivity are the main keywords that inform the designers' work, their practices for systems development and the very (social) 'nature' of technology in contemporary society.

Therefore, the aim is to portray, accordingly with Iacono and Kling's analysis of computerization movements [2001], some of the macro-social and cultural forces involved in the emergence of ubiquitous and mobile computing as the next wave in computerization development and specific sub-culture in computerization movements. In fact, the metaphor of ubiquity provides a key-access to discursive and organizational practices of inclusion/exclusion linked with the emergence of a mobile Internetworking, a mobile society, and the everyday life 'on the move'.

The arguments presented in this contribution also aim to draw a starting theoretical framework to enquire intersections at crossroads of mobility, technologies, everyday life and different social groups using mobile devices in their work and non-work everyday life.

\section{Mediatized everyday life}

The everyday life of individuals and organizations is increasingly mediated by various types of technological artefacts aimed to share information and allow communication at a distance. These artefacts are more and more embedded in the texture of everyday life, in at least two senses.

First and foremost, this embeddedness passes through a process of 'naturalization' of artefacts, which renders them invisible and transparent to the user's attention and sight. This process, named as 'domestication' [Silverstone, 1994] constitutes the very essence of contemporary everyday life: what we progressively take for granted, among other things, is the routine to cope with always new technological artefacts.

Silverstone provided various examples of domestication of television as both an object and a medium, but any new technology, at its beginning, requires to be domesticated, appropriated by users, through various strategies. Everyday life as more and more mediatized, that means mediated and shaped by Information and Communication Technologies, coincides, among other things, with the routinization of multiple sociotechnical innovations.

Secondly, there is an even more materialistic aspect in the process of embedding technology and rendering it invisible: literally, technology 'disappears', o 'hides itself', in our pocket, hand, body and environment. This is what is called 'ubiquitous' computing, that means the encounter between mobile and pervasive computing, which associates computing with a high degree of both mobility and embeddedness in the fabric of everyday life [Lyytinen and Yoo, 2002a]. Nomadic information environments are said to move towards "ubiquitous computing, in which computers will be embedded in our natural movements and interactions with our environments both physical and social. Ubiquitous computing will help organize and mediate social interactions wherever and whenever these situations might occur" [Lyytinen 
and Yoo, 2002b: 63]. In fact, increased physical and virtual mobility is fostered and enhanced by mobile and ubiquitous computing environments, which blur boundaries between work and non-work settings and contribute to make everyday life of individuals and organizations more and more mediatized. These technologies are taken for granted more easily than others, as they tend quite literally to disappear in our pockets or hands. Invisibility [Weiser, 1994] is the metaphor used to describe this type of technologies: again such a metaphor fits in the concept of everyday life and shared meaning based on taking for granted what is defined as 'reality' from a phenomenological approach and constructivist viewpoint [Berger and Luckmann, 1967]. Furthermore, invisibility and miniaturization contribute to make sociotechnical relationships more complicated, fragile and complex as technologies are pervasively embodied in sociotechnical environments. Awareness of technology use and its participation to daily action and interaction are partly masked and weakened by this embodiment. New (dis)continuities in space and time, and in (mobile) everyday life emerge.

As a consequence, technology is not simply (and not anymore) 'out there': even before being domesticated by social groups who appropriate it into their own culture, technology is invisible, or less visible than it was before. Far from being a trivial aspect of the question, the increased invisibility of technological artefacts, their miniaturization and portability re-frame in a relevant way social use and perception of technology in everyday life. New configurations emerge along the sociotechnical continuum of the conception, development and use of technological systems and the social construction of them. Adhering to the stream of a clear anti-deterministic approach to technology, the analysis of the social and imaginary genealogy of these sociotechnical configurations becomes crucial. Such a hot issue - of not seeing technology as separated from its constitutive 'sociality' or 'socialness' - can be faced with tools and concepts provided by Social Informatics as devoted to examine social aspects of computerization.

"Social Informatics studies aim to ensure that technical research agendas and system designs are relevant to people's lives. The key word is relevance, ensuring that technical work is socially-driven rather than technology-driven" [http://rkcsi.indiana.edu/article.php/about-social-informatics/35]. Social-ness does not constitute a separate requirement or dimension in/of computerization, instead shaping it since the very beginning.

\section{Discursive frames around ubiquity: a set of 'affiliated concepts'}

The main reference in this paper is constituted by Iacono and Kling's analysis of computerization movements [2001] as providing a clear theoretical framework to understand mobile systems development and the emergence of mobile technological devices These phenomena are framed here in the context of the mobility and mobilities paradigm [Urry, 2003] but also as current manifestation (a kind of subculture) of computerization movements. By referring to the model of public discourse and discursive frames as alternative conceptualization of the 
Internetworking, it will be possible to understand how macro social discursive frames are enacted to make sense of an increasing number of technological artefacts (e.g. wifi architectures, smart mobile phones, PDAs, I-pods and so on).

Iacono and Kling [2001] provided us with an in depth analysis of the Internetworking and distant forms of work as sociotechnical arrays emerging from the joint action of computerization movements, rather than from mere economic or technical factors. They put together the emergence of a new technological architecture (Internetworking) and the societal issues framed in layered discursive practices, which constituted the outcome and inspiration of computerization movements' action.

"(...) Participants in computerization movements build up frames in their public discourses that indicate favourable links between Internetworking and a new preferred social order. These frames help to legitimate relatively high levels of investment for many potential adopters and package expectations about how they should envision a future based on Internetworking" [Iacono and Kling, 2001: 97]. Iacono and Kling identified three components to explain at a theoretical level how macro-social and cultural components become part of the process of societal mobilization that makes the Internetworking (or any other technology, e.g. the mobile) work and develop. The three components are technological action frames, public discourse and organizational practices. "These three elements (...) are related (...) Technological action frames shape and structure public discourse whereas public discourse shapes and structures organizational practices (...) But these relationships are nondeterministic (...) Relationships among the three elements also can be recursive. People may enrich their discourses and even modify their frames as they struggle to discuss the actual complexity of their practices. As a consequence, practices can generate new discourses, and new discourses can build up new technological frames"' [Iacono and Kling, 2001: 100-101].

Universal access, death of distance, obliteration of time and space, totalizing rhetoric of progress and belief in a new (better) social order: around these topoi, the computerization movements built up their idea of Internetworking. It is noticeable that all of these concepts evoke and are evoked again in the idea of ubiquity and pervasivity associated with the rise of mobile Internetworking and ubiquitous computing. What wifi architectures and mobile computing devices add to this rhetoric is the belief in an almost totally invisible, transparent and embedded computing network: the disappearance of any materiality of Internetworking and hardware architectures, to the extent of their total 'softening'. Here ubiquity suggests the capacity and ability to indefinitely navigate into invisible spaces that annihilate time, space and (potentially), any other difference.

Around ubiquity, a set of 'affiliated concepts' are built up to reinforce the development and adoption of mobile (ubiquitous) technologies: miniaturization, pervasivity, wearability, portability and, of course, mobility. All of them contribute to draw a complex picture of the new technology: they are the 'bricks of meaning', which constitute the technological action frame for ubiquitous computing, and they also pervade the different types and sources of public discourse about ubiquitous technologies. In fact, "technological action frames circulate in public discourses and act as a form of currency whose structure and meaning remain relatively constant across a variety of discursive practices" [Iacono and Kling, 2001: 110-111]. 
Iacono and Kling identified four layers of public discourse: government discourse, scientific discipline discourse, mass-media discourse, professional and institutional discourse [2001]. Combinations and hybridations among these layers can be traced: for example, mass-media discourse is the most pervasive as it feeds and amplifies the other layers of public discourse.

Also, to some extent we could observe overlaps between professional and scientific discourses, especially with reference to the design and development of mobile and ubiquitous computing systems (see par. 4.1).

Mass-media and professional discourse will be shortly analyzed with reference to ubiquitous technology in this paper. Of the concepts constituting the technological frame for this technology, mobility will be analyzed in particular, as key-carrier of meaning. In fact, all the other affiliated concepts and frames can be understood and highlighted as a continuous re-call to mobility and ubiquity, both enabled and enhanced by mobile technological artefacts.

\section{Mobilizing support for mobility and ubiquity}

Contemporary Western societies can be depicted, among other things, as affected by what Urry [2003] calls 'compulsion to mobility'. Such a compulsion comprises representations of travel (of both people and cultures) as well as the idea of a contemporary citizen 'on the move'.

For the aim of this paper it is crucial to link the trend towards an increased mobility ['hypermobility,' cf. Urry, 2002] to the trend towards an increased 'mediatization' [hypermediatization, cf. Pellegrino, 2004] of organizational and social life, at both macro and micro context level. The intersection between hypermobility and hypermediatization - what I call 'mediatized mobility' - is of particular interest as discursive frame to understand current transformations in the paradigm of Internetworking.

“(...) People in prosperous industrialised societies are both increasingly on the move and communicating more to reach and connect with absent others. Thus developments in transport and communication technologies not merely service or connect people but reconfigure social networks by disconnecting and reconnecting them in complex ways. Thus as easy availability of cars, trains, planes and communication technologies spread social networks beyond cities, regions and nations, so they reconnect them by helping to afford intermittent visits, meetings and communication at-a-distance. People can travel, relocate and migrate and yet still be connected with friends and family members 'back home' and elsewhere. So increasingly, people that are near emotionally may be geographically very far away; yet they are only a journey, email or a phone call away" [Larsen et al., 2005: 10].

Intermittent and at-a-distance communications are part of both ubiquity and pervasivity: they are not simply based on the overcoming/obliteration of distances in time and space as in the rise of Internetworking [Iacono and Kling, 2001] but on their restructuration, based on mixed forms of co-presence, travel and mobilities.

"Indeed all forms of social life involve striking combinations of proximity and distance, combinations that necessitate examination of the intersecting forms of 
physical, object, imaginative and virtual mobility that contingently and complexly link people in patterns of obligation, desire and commitment, increasingly over geographical distances of great length" [Urry, 2002: 1].

The idea of a mobile society and of interconnected, different 'mobilities' on which this society is structured [Urry, 2002; Larsen et al., 2005] can be identified as the main framework that feeds and reinforces the technological frame for ubiquitous technologies. In this respect, emphasis on miniaturization and portability is strongly associated with a perceived increase of travel. Travelling in the information age means new and different things: there is a corporeal travel, which does not either end or exhaust the possibilities of mobility. Mobilities of objects and information do accompany and transform corporeal travel. The combination of instrumental means of communication and humans brings about, while replacing "the spatiality of "copresent sociality', new modes of objectified stranger-ness" [Urry, 2002: 7]. The idea of ubiquity resides in this shift from a co-presence based on physical proximity to being 'anywhere anytime' through a combination of distance and proximity increasingly enabled and supported by mobile devices.

Dispersed symptoms and traces of a societal mobilization towards mobility are in the air and start to widespread; similarly to the death of distance and the rhetoric of a new social order based on Internetworking, the mobile Internet has its founding framework into the perspective (and the current reality) of a mobile society and of citizens whose everyday life is more and more 'on the move'.

Personal media and connectivity through mobile and ubiquitous computing emphasize the individual-ness of mobile devices. By contrast, references to a strong constitutive social dimension and the re-discovery of a community sense (and of local-ness associated with informational networks) pervade some of the literature on ubiquitous and mobile devices, labelled as 'mobile social software' [cf. Melinger, 2004]. Such references go back to the utopian terms through which new technological systems and infrastructures are described: in the rhetoric, Internetworking is "more direct, participative, democratic, socially engaging, and community oriented" than any other technological infrastructure for communication [Iacono and Kling, 2001: 113].

Furthermore, not only are people mobile, but also cultures can be described as 'on the move' (Urry, 2003). In this respect, the increasing technological mediation of everyday life draws both continuities and discontinuities with previous and current mobilities.

On the one hand, the dis-anchorage from space and time enabled by mobile communication reinforces the perception of ubiquity as "appropriate for an examination of mobile communication. The device is seemingly ubiquitous in its diffusion and in its role as the Swiss army knife of personal technologies" [Ling, 2004: 5].

On the other hand, such a ubiquity, associated with a pervasive diffusion of mobile phones, is very flexible and can be contextualised differently: an example of the 'context-sensitivity' of mobile technologies and artefacts is provided by the possible combination among what Larsen et al. (2005) define 'different mobilities' and the fact that "the mobile phone with SMS text is enabling the flexibilisation of people' s path through time-space (...)" [Urry, 2002: 8]. 
Different and interdependent mobilities (of people physically travelling, of objects, of information, images and memories, virtual travel on the Internet and communicative-mediated travel; Urry, 2000 and 2003) are differently supported by communicative and technological mediation, with striking combination of mobility and 'immobility', as in the use of ubiquitous computing in the household environment and the renewed emphasis on the home as privileged target for the consumer electronics and media fruition (e.g. attempts to integrate the Internet and television). Furthermore, "family life is becoming plugged into an ever-expanding array of communication technologies that connect families to one another and to the outside world often at great distance. Lives are rarely if ever "local" " [Larsen et al., 2005: 5].

Then there are (at least) two sides in the discourse of mobility and in the emergence of mobile and ubiquitous technologies. The combination of hypermobility and hypermediatization can result into an increased physical and virtual mobility. Being mobile or not, mobile devices let us afford different kinds of travelling in the information age, recalling what Williams (1974) observed within the diffusion of automobiles and television: a restructured 'mobile privatization', moving towards what we define mediatized, individual and connected mobility.

At this stage, we need to move towards the link between discursive practices about mobility and mobile technological artefacts.

\section{From ideas to artefacts (and the other way round): back to location?}

The three folded model of Iacono and Kling [2001], based on the analytical distinction among technological frames, public discourse and organizational practices, fits the attempt to understand how ideas performed in public discourse come to be translated into technological artefacts: according to Bijker and the SCOT approach [Bijker, 1995], technologies are characterized by interpretative flexibility and constituted as different by relevant social groups who interpret and make sense of them. What is of particular interest with reference to mobile and ubiquitous technology is that these interpretations are totally 'in the making' and they start to draw a path paved of various discursive frames, aimed to establish some favourable links between technological-based networks and social networks and communities. A joint analysis of continuities and break-downs with reference to ideas and artefacts can be drawn in order to understand how and when artefacts come to embed (and support) discursive practices established 'around' them.

"People are able to 'plug into' global networks of information through which they can 'do' to at least certain objects (especially with increased bandwidth) and 'talk' to people without being present in any particular place, without their bodies having to travel. 'Persons' thus occur through various nodes in these multiple networks of communication and mobility. Their body's corporeal location is less relevant in these networks of person-person communication" [Urry, 2002: 8].

This progressive distanciation or separation between physical location and networked communication is one side of mobile and ubiquitous computing. "Mobile 
phone cultures generate small worlds of perpetual catching up and small talk on the move, blurring distinctions between presence and absence" [Larsen et al., 2005: 50]. On other hand, these small worlds can be supported or enforced by the so called 'location aware technologies' based on specific uses of the mobile infrastructure [cf. www.socialight.net/edu]. These technologies seem to re-orient and anchorage mobility back to physical spaces and location. Design and development of mobilesystems/architectures, in this respect, are exemplary settings for tracing discursive frames linked with local organizational practices. A short review of discourses emerging in the scientific disciplines, professional communities and mass-media advertisement of new mobile services will be proposed in the next section.

\section{The scientific/professional discourse: Technology-driven vs mobile social software perspective}

In what direction are discourses and practices on mobile systems and technologies evolving? As discourses are coherent or contradictory traces of embedded organizational practices, it is of the greatest importance to retrieve and retrace discourses in order to understand the most 'local' level in the emergence of new technologies. In this respect, different perspectives of discourse can be identified, layered in the scientific research arena, professional communities and mass-media. It is very important to note that in the discourse about technological infrastructures and devices in general, and about mobile systems in particular, boundaries between scientists, technologists and professional communities linked with specific industries are blurred enough to say that designing mobile and ubiquitous computing systems results from interwoven discursive and organizational practices.

In their review of 105 articles concerning mobile systems development, Hosbond and Jensen [2005] retrieved four perspectives: the 'requirement' perspective, the 'technology' perspective, the 'application' perspective and the 'business' perspective. They conclude noting "the mobility debate so far has been largely technology-driven. The strong focus on technology is an obvious indicator of continuous development and innovation within this field, which reflects somewhat immature technologies, but also emphasizes a strong demand for more robust and flexible technologies" [Hosbond and Jensen, 2005: 11].

On the level of more specific organizational practices, a counter discourse can be retrieved and identified, addressing community and social networking issues rather than the individualizing and personalizing side of mobile and ubiquitous computing. This is "the field of MoSoSo (Mobile Social Software)", which "is evolving quickly, with many new types of applications being developed and deployed. MoSoSo at its most basic level is any software that allows people to connect with others while mobile" [Melinger, 2004a: 1]. This comprises, for example, "a platform that allows people to connect with others in their social network by using mobile telephone handsets in novel ways. Using the current or past location of friends, the (...) platform provides a number of subtle and overt tools which enable unique modes of real-time and time-shifted communication" [http://socialight.net/edu/what.htm]. 
On other hand, ubiquitous computing goes farther than mobile as "the main challenges in ubiquitous computing originate from integrating large-scale mobility with the pervasive computing functionality. In its ultimate form, ubiquitous computing means any computing device, while moving with us, can build incrementally dynamic models of its various environments and configure its services accordingly. Furthermore, the devices will be able to either 'remember' past environments they operated in, thus helping us to work when we re-enter, or proactively build up services in new environments whenever we enter them" [Lyytinen and Yoo, 2002: 64)]. Such a flexible and 'shapeless' infrastructure, then, will be totally surrounding the everyday life environments and constitutive of them.

However, emphasis on technology rather than society as a recurrent frame of public discourse is not new, neither it is totally new the role played by mass-media in framing the mobile world, society and Internetworking.

Mass-media imaginary and discourse about the mobile world, in fact, reveal a powerful set of stereotypes in delivering the added value of mobile services as devoted to support the individual in his/her social life, to personalize his/her social networks, to bring the world 'around' in the pockets/hands as an extended body. Emphasis on miniaturization and portability of technological devices constitutes a leitmotiv of this discourse, which feeds both the layers of public discourse identified (the technology-driven level and the social software level).

In this respect, the Italian context is very interesting with reference not only to the levels of mobile phone diffusion, but also with the symbolic and marketed-toconsumer struggle to enrol new users and expand quality and quantity of mobile services (e.g. TV advertisement especially).

The layers of discourse shortly examined show, on the one hand, a big emphasis on technology as the key-carrier of meaning in practices of mobile systems development (Hosbond and Nielsen, 2005) and, on the other hand, a trend to go back to physical location and afford the re-construction of social connectedness at $-\mathrm{a}$ distance, as in the discourse of mobile social software (Melinger, 2004a and 2004b) This discourse, however, could even be framed as a masked version (ideologically informed) of the technology-driven approach, as it subtly suggests that sociality and community are direct 'emanations' of the mobile software.

\section{Politics of signification, inclusion and exclusion as human choice in computerization}

"Both the optimistic and the pessimistic stories of computerization are often crafted with the conventions of utopian and anti-utopian writing. The utopian and antiutopian genres of social analysis are about 500 years old, and predate the social sciences by about 350 years. Authors who write within these genres examine certain kinds of social possibilities, and usually extrapolate beyond contemporary technologies and social relationships. Utopian tales are devised to stimulate hope in future possibilities as well as actions to devise a self-fulfilling prophecy. In contrast, anti-utopian tales are devised to stimulate anger at horrible possibilities and actions to avoid disaster" [Kling, 1996: chapter A section II]. 
Horrors and hopes can be traced in any technological imagery and public discourse about technologies, in both older and newer media. These attitudes express fear and passion towards embedding a social perspective in narratives of technological development: social expectations framed in public discourse are part of the politics of signification carried out and performed by what we could call, with Iacono and Kling [2001] as 'computerization movement activists', or, with Bijker [1995], 'relevant social groups acting in a specific technological frame and constituting it'. Therein, a social informatics perspective deals necessarily with politics of signification: in these politics patterns of action and issues linked with the role of human choice in computerization can be discovered and identified. Mobile and ubiquitous technologies seem to suggest, as in the Internetworking rhetoric [Iacono and Kling, 2001] the utopia of a universal mobile society, where travelling and intermittent co-presence are taken for granted, routinized patterns of action.

However, mobility is neither always a free choice nor is travel 'a fundamental human right' [Kaplan cit. in Urry, 2003] in itself and for all.

Mobility and, correspondingly, immobility, can be coerced or freely chosen (Urry, 2002) and at the same time a 'mobile divide' can be drawn from the apparent hypermobility of contemporary societies, with different possibilities to access and experience mobilities in their mediatized and not-mediatized face.

Who is entitled to mediatized mobility is, often, member of micro communities, or 'mobile elites'. Different communities can aggregate themselves around socioinformational networks where they share specific technological artefacts, services and uses of technologies. These can be variously determined as brand communities (e.g. I-pod users) or loosing-coupled networks of users sharing a common communication model embedded into a specific protocol (e.g. Bluetooth users). Despite the diffusion of the mobile phone and its widespread popularity (now starting and quickly raising in developing countries as new privileged markets for mobile services companies and providers), paths of exclusion and inclusion can be traced at both the level of macro technological infrastructures or architectures, and at the level of e-literacy in using smaller and multifunctional technological devices. On the two sides, different paces and degrees in appropriating technology can be observed. Furthermore, privacy issues seem to constitute the 'horrific' side of mobile and ubiquitous technologies, with the re-discovery of risks of total surveillance and traceability of the 'electronic body'. Permanent connectivity and availability, associated with recording of conversations and messaging under the urgency (and excuse) of international terrorism after September $11^{\text {th }}$ exasperated the emphasis on control and monitoring of communication at a distance. This happens even more with mobile technologies, as "new types of location-aware mobile social software (MoSoSo) applications allow people to develop both weak and strong relationships with others who live and work around them (...) However, location aware MoSoSo applications can bring with them the ability to closely monitor community members and invite unwanted communications, characteristics that often prompt anxieties about intrusions into privacy" [Melinger, 2004b: 1].

In this respect, ubiquity as associated with technologies anywhere anytime for everybody hides the (forced) inclusionary path into an invisible network of global surveillance, but also the exclusionary paths which seclude people and communities to coerced (im)mobility and dependence from a thick, seamless web of relations in 
proximity and distance, or proximity at distance. As Iacono and Kling [2001: 130] concluded, "in their most likely form, the computerization movements of this new century will constitute a conservative transformation reinforcing patterns of an elitedominated, stratified society". Human choice in computerization has to do with inclusionary and exclusionary politics, performed at different levels. On the level of meanings and symbolic struggles, contending discursive frames orient the miniaturization and portability of technological devices towards individualized or community oriented uses. Discursive frames elaborated in the setting of scientific and professional discourse, as well as in the media, on other hand, contribute to shape ubiquitous technological artefacts in specific directions, to the extent that the mobile phone as object becomes a symbol of fashionable life styles and a medium open towards meaningful practices of consumption and appropriation (cf. Silverstone, 1994) : as it incorporates shared meanings in a specific shape, it can be truly considered a 'cultural object' (Griswold, 1994).

Entering these frames means to recover the mundane root of technological artefacts cultivated and growing through a sociotechnical, 'fully social driven' process. Such a project, we think and claim, is coherent with Kling's constant attention to societal mobilization around technologies.

\section{Conclusions}

The imperative of mobility and increasing embeddedness of artefacts in the material and immaterial fabric of everyday life were analyzed in this contribution as the starting points to understand mediatization and mobility characterizing contemporary society. Ubiquity as a metaphor and perspective for development of new technological artefacts was de-constructed through the analysis of the technological frame of mobile and ubiquitous computing. Such a frame comprises discursive practices about mobility, ubiquity, miniaturization and other affiliated concepts, which overall depict the 'imaginary in the making' of the 'mobile Internetworking' as a specific sub-culture in computerization movements (cf. Iacono and Kling, 2001). From this analysis, different contending discourses emerged: ubiquity as technology anywhere, anytime, for everyone unravels its ideological veil in hiding practices of inclusion and exclusion carried out through politics of signification; both indifference to and awareness of physical location constitute directions for development of mobile systems and architectures; proximity and distance, presence and absence are evoked and enabled by mobile and ubiquitous computing. The way domestication of artefacts proceed is far from indifferent to all this: societal mobilization around technologies always tells us what those technologies will (not) become and, especially, to whom they will (not) deliver their horrors and hopes, beliefs and images, constraints and opportunities. 


\section{References}

1. P. Berger, and T. Luckmann, The social construction of reality (Doubleday, New York, 1967).

2. W.E Bijker, Of Bycicles, Bakelites and Bulbs (The MIT Press, Cambridge, 1995).

3. W. Griswold, Cultures and Societies in a Changing World (Pine Forge Press, USA London New Dheli, 1994).

4. S. Iacono and R. Kling, in: Information Technology and Organizational Transformation. History, Rhetoric and Practice, edited by: J. Yates and J. Van Maanen (Sage Publications, Thousand Oaks:, 2001), pp. 93-135.

5. R. Kling, (ed.), Computerization and Controversy. Value Conflicts and Social Choices (Academic Press, San Diego, 1996, $2^{\text {nd }}$ edition).

6. R. Kling, Center for Social Informatics, History of the term (October 10, 2005); http://rkcsi.indiana.edu/article.php/about-social-informatics/35

7. R. Ling, The Mobile Connection. The Cell Phone's Impact on Society (Morgan Kauffman Publishers, an Imprint of Elsevier, San Francisco CA, 2004).

8. J. Larsen, J. Urry, and J. Axhausen, Social Networks and Future Mobilities. Report to the UK Department for Transport. Final draft, December 2005; http://www.lancs.ac.uk/fss/sociology/cemore/cemorepublications.htm

9. K. Lyyttinen, and Y. Yoo, Research Commentary: The Next Wave of Nomadic Computing, Information Systems Research, 13(4), 377-388 (2002a).

10. K. Lyyttinen, and Y. Yoo, Issues and Challenges in Ubiquitous Computing, Communications of the ACM, 45(12), 63-65 (2002b).

11. D. Melinger, Privacy's role in Mobile Social Software for the Urban Community, Paper presented at the 6th International Conference on Ubiquitous Computing, Nottingham, 7-10 September (2004a).

12. D. Melinger, Privacy and Community in the Design of Mobile Social Software, Paper presented at the International Conference on Mobile Communication, Seoul, 18-19 October (2004b).

13. G. Pellegrino, Hypermediatization and Inertia: Patterns of Technological Appropriation between Reproduction and Innovation, Paper presented at the $20^{\text {th }}$ Egos Colloquiuum, Ljubljana, 3-5 July (2004); www.egosnet.org (member area).

14. R. Silverstone, Television and Everyday Life (Routledge, London, 1994).

15. Socialight (2004); http://socialight.net/edu/

16. Socialight, What is Socialight (2004); http://socialight.net/edu/what.html

17. J. Urry, Sociology beyond Society: Mobilities for the $21^{\text {st }}$ Century (Routledge, London, 2000).

18. J. Urry, Mobility and Proximity, Sociology, 36(2) 255-274 (2002), retrieved in http://www.ville-en-mouvement.com/interventions/John_Urry.pdf

19. J . Urry, Mobile Cultures, Paper published by the Department of Sociology, Lancaster University, at http://www.comp.lancs.ac.uk/sociology/papers/UrryMobile-Cultures.pdf (2003).

20. M. Weiser, The world is not a desktop, Interactions, January, 7-8 (1994).

21. R. Williams, Television: Technology and Cultural Form (Fontana, London, 1974). 